\title{
FINITE ELEMENT MODEL VALIDATION OF THE HYBRID-III RAIL SAFETY (H3-RS) ANTHROPOMORPHIC TEST DEVICE (ATD)
}

\author{
Shaun Eshraghi \\ Volpe National Transportation Systems Center \\ Cambridge, MA, USA \\ David Hynd \\ Transport Research Laboratory \\ Wokingham, Berkshire, UK
}

\section{ABSTRACT}

The Hybrid-III Rail Safety (H3-RS) anthropomorphic test device (ATD), also known as a crash test dummy, was developed by the Rail Safety and Standards Board (RSSB), DeltaRail (now Resonate Group Ltd.), and the Transport Research Laboratory (TRL) in the United Kingdom between 2002 and 2005 for passenger rail safety applications [1]. The H3-RS is a modification of the standard Hybrid-III 50th percentile male (H3-50M) ATD with additional features in the chest and abdomen to increase its biofidelity and eight sensors to measure deflection. The H3-RS features bilateral (left and right) deflection sensors in the upper and lower chest and in the upper and lower abdomen; whereas, the standard H3-50M only features a single unilateral (center) deflection sensor in the chest with no deflection sensors located in the abdomen.

Additional H3-RS research was performed by the Volpe National Transportation Systems Center (Volpe Center) under the direction of the U.S. Department of Transportation, Federal Railroad Administration (FRA) Office of Research, Development, and Technology. The Volpe Center contracted with TRL to conduct a series of dynamic pendulum impact tests [2]. The goal of testing the abdomen response of the H3-RS ATD was to develop data to refine an abdomen design that produces biofidelic and repeatable results under various impact conditions with respect to impactor geometry, vertical impact height, and velocity.

In this study, the abdominal response of the H3-RS finite element (FE) model that TRL developed is validated using the results from pendulum impact tests [2]. Results from the pendulum impact tests and corresponding H3-RS FE simulations are compared using the longitudinal relative deflection measurements from the internal sensors in the chest and abdomen as well as the longitudinal accelerometer readings from the impactor. The abdominal response of the H3-RS FE model correlated well with the physical ATD as the impactor geometry,

\author{
Kristine Severson \\ Volpe National Transportation Systems Center \\ Cambridge, MA, USA \\ A. Benjamin Perlman \\ Volpe National Transportation Systems Center \\ Cambridge, MA, USA
}

vertical impact height, and velocity were changed. There were limitations with lumbar positioning of the H3-RS FE model as well as the material definition for the relaxation rate of the foam in the abdomen that can be improved in future work.

The main goal of validating the abdominal response of the dummy model is to enable its use in assessing injury potential in dynamic sled testing of crashworthy workstation tables, the results of which are presented in a companion paper [3]. The authors used the model of the H3-RS ATD to study the 8G sled test specified in the American Public Transportation Association (APTA) workstation table safety standard [4]. The 8G sled test is intended to simulate the longitudinal crash accleration in a severe train-to-train collision involving U.S. passenger equipment. Analyses of the dynamic sled test are useful for studying the sensitivity of the sled test to factors such as table height, table force-crush behavior, seat pitch, etc., which help to inform discussions on revisions to the test requirements eventually leading to safer seating environments for passengers.

\section{INTRODUCTION}

Impacts with thin, rigid workstation tables have been identified as a serious safety concern during train accidents $[5,6$, $7,8]$. Workstation table safety standards have been adopted by industry groups such as APTA [4] and the RSSB [9]. These safety standards set performance requirements for energyabsorbing tables that are designed to lower the risk of serious injury to the chest and abdomen in an accident. Injury criteria are established in the table standards so that occupants seated at tables should experience roughly the same level of protection as occupants seated in forward facing row-to-row seating.

FE analysis can be used to evaluate the risk of injury from a given table design in an accident with a validated FE model of an ATD that has a biofidelic chest and abdomen response. The THOR-50M and H3-RS are advanced ATDs that have been developed to have biofidelic chest and abdomen responses based

This work was authored in part by a U.S. Government employee in the scope of his/her employment.

ASME disclaims all interest in the U.S. Government's contribution. 
on post-mortem human subject (PMHS) testing [10, 11]. The National Highway Traffic Safety Administration (NHTSA) and the University of Virginia validated the FE model of the THOR50M ATD chest and abdomen using three pendulum impact tests [12].

In this study the abdomen response of the H3-RS FE model, developed by TRL, is validated using pendulum impact test results reported previously [2]. The abdomen response of the H3RS FE model is being validated to study dynamic impacts with an energy-absorbing table design which is presented in a companion paper [3]. This research is being conducted to inform decisions regarding performance requirements in workstation table safety standards, with the goal of minimizing accident consequences from table impacts and increasing rail safety.

\section{METHODS}

TRL loaned version 2.2 of the H3-RS FE model in LSDYNA [13] to the Volpe Center to validate the chest and abdomen response of the model and make changes as needed. The H3-RS FE model is a substantial modification of the H350M FE model that was jointly developed by Livermore Software and Technology Corp. (LSTC) and the National Crash Analysis Center (NCAC) at George Washington University and is freely available from LSTC's website.

The H3-RS is composed of the head, shoulder, arm, upper thorax, and lower leg assemblies from the H3-50M, and the lumbar spine, pelvis, and femur assemblies are from a previous version of the THOR-50M. The H3-RS features custom modifications of the abdomen assembly which includes an abdomen bag that extends $20 \mathrm{~mm}$ upwards into the rib assembly. The tie between the ribs and abdomen prevents impacting objects from penetrating the space between lower ribs and upper abdomen which is a concern for the standard H3-50M.

In the H3-RS, chest deflection is measured using bilateral compact rotary unit (CRUX) sensors in the upper and lower chest. The upper abdomen deflection is measured with bilateral double-gimballed string potentiometers (DGSPs), and the lower abdomen deflection is measured with bilateral string potentiometers (SPs).

The H3-RS FE model was positioned on a rigid flat bench using LS-PrePost ${ }^{\circledR}$ V4.2 and settled using a gravity step of 75 ms according to the procedure outlined in the H3-50M FE model user manual [14]. A settling step was used to capture the lumbar positioning observed in testing and the deformation of the pelvis foam.

Automatic surface-to-surface contact definitions were specified with static and sliding coefficients of friction of 0.2 between the ATD and the rigid bench and the rigid impactor. The H3-RS FE model has a lumbar spine similar to the THOR-50M that is locked, making it difficult to capture the lumbar positioning of the physical ATD in the model. A comparison of the dummy positioning is shown in Figure 1 and Figure 2 using the lower abdomen repeatability test (Test \#7) as an example.



Figure 1. Lower Abdomen Repeatability Test Setup (Tests \#3, \#7, \#8)



Figure 2. Lower Abdomen Repeatability Test FE Model

Rigid FE models were also created for the five impactor designs used in testing: (1) $26.7 \mathrm{~mm}$ diameter, $18 \mathrm{~kg}$ steering wheel; (2) 25 mm diameter, $32 \mathrm{~kg}$ round bar; (3) $25.4 \mathrm{~mm}, 18 \mathrm{~kg}$ round bar; (4) $50 \mathrm{~mm}, 18 \mathrm{~kg}$ round bar; (5) $50.8 \mathrm{~mm}, 32 \mathrm{~kg}$ round bar. For each simulation, the bench and impactor were constrained in all degrees of freedom except longitudinally for the impactor. A longitudinal initial velocity corresponding to the pendulum impact velocity was assigned to the impactor. An automatic surface-to-surface contact definition was applied to the exterior shell elements of the ATD and the rigid impactor. The simulations terminated at $60 \mathrm{~ms}$ with automatic mass-scaling and a target time step of 5.55e-4 ms.

Out of 27 pendulum impact tests conducted on the physical H3-RS ATD, 16 were selected to validate the abdomen response of the H3-RS FE model. Eleven tests were excluded because they either used an alternative abdomen bag design or the impactor diameter was too small to be representative of energyabsorbing table designs. The aggregated test matrix is given in Table 1. 
Test 7 is based on PMHS testing reported by Cavanaugh et al. [10] in 1986 which is used in the lower abdomen certification (BioDB [15] Test No. 11580) of the THOR-50M ATD [12]. Test 9 is based on PMHS testing reported by Nusholtz and Kaiker [11] in 1994 which is used in the upper abdomen certification (BioDB Test No. 11388) of the THOR50M ATD. Tests 7 and 9 were conducted three times each to study their repeatability. The remaining tests with the large diameter $18 \mathrm{~kg}$ and $32 \mathrm{~kg} 50 \mathrm{~mm}$ round bars were each conducted once.

Table 1. Aggregated test matrix

\begin{tabular}{cccccc}
\hline $\begin{array}{c}\text { Test } \\
\text { Number }\end{array}$ & $\begin{array}{c}\text { Vertical Impact } \\
\text { Height (mm) }\end{array}$ & $\begin{array}{c}\text { Impactor } \\
\text { Diameter }(\mathrm{mm})\end{array}$ & $\begin{array}{c}\text { Impactor } \\
\text { Mass }(\mathrm{kg})\end{array}$ & $\begin{array}{c}\text { Target Velocity } \\
\left(\mathrm{m} \cdot \mathrm{s}^{-1}\right)\end{array}$ & $\begin{array}{c}\text { Impact Velocity } \\
\left(\mathrm{m}^{-1} \mathrm{~s}^{-1}\right.\end{array}$ \\
\hline$\# 1$ & DGSP +15 & $26.7^{\ddagger}$ & 18 & 8.0 & 7.98 \\
$\# 2$ & SP +0 & 25.0 & 32 & 6.1 & 6.13 \\
$\# 3$ & SP +0 & 25.0 & 32 & 6.1 & 6.12 \\
$\# 4$ & DGSP +15 & $26.7^{\ddagger}$ & 18 & 8.0 & 7.97 \\
$\# 5$ & DGSP +15 & $26.7^{\ddagger}$ & 18 & 8.0 & 7.98 \\
$\# 6$ & SP +0 & 25.0 & 32 & 6.1 & 6.11 \\
$\# 7$ & SP +0 & 25.0 & 32 & 6.1 & 6.11 \\
$\# 8$ & SP +0 & 25.0 & 32 & 6.1 & 6.11 \\
$\# 9$ & DGSP +15 & $26.7^{\ddagger}$ & 18 & 8.0 & 7.99 \\
$\# 10$ & DGSP +15 & $26.7^{\ddagger}$ & 18 & 8.0 & 7.99 \\
$\# 11$ & DGSP +15 & 25.4 & 18 & 8.0 & 7.98 \\
$\# 12$ & DGSP +40 & 25.4 & 18 & 8.0 & 7.99 \\
$\# 13$ & DGSP -10 & 25.4 & 18 & 8.0 & 7.97 \\
$\# 14$ & SP +25 & 25.0 & 32 & 6.1 & 6.11 \\
$\# 15$ & DGSP +0 & 25.4 & 18 & 8.0 & 7.99 \\
$\# 16$ & DGSP +0 & 50.0 & 18 & 8.0 & 8.04 \\
$\# 17 a$ & DGSP +15 & 50.0 & 18 & 8.0 & 8.05 \\
$\# 18$ & DGSP +40 & 50.0 & 18 & 8.0 & 8.03 \\
$\# 19$ & DGSP -35 & 25.4 & 18 & 8.0 & 8.00 \\
$\# 20$ & DGSP -35 & 25.4 & 18 & 8.0 & 7.99 \\
$\# 21$ & DGSP -10 & 50.0 & 18 & 8.0 & 8.03 \\
$\# 22$ & SP +0 & 50.8 & 32 & 6.1 & 6.12 \\
$\# 23$ & SP +25 & 50.8 & 32 & 6.1 & 6.12 \\
$\# 24$ & DGSP +15 & 50.0 & 18 & 6.0 & 6.02 \\
$\# 25$ & DGSP +15 & 50.0 & 18 & 7.0 & 6.98 \\
$\# 26$ & SP +0 & 50.8 & 32 & 5.1 & 5.15 \\
& & & & & \\
\hline
\end{tabular}

(1) Only test entries in grey used for model validation; (2) Positioning is vertical distance in millimeters from DGSP (upper abdomen) or SP (lower abdomen) sensors; (3) ${ }^{\ddagger}$ denotes Steering Wheel geometry

The following measurements were used to compare simulation results with test data: (1) longitudinal impactor force (calculated using pendulum accelerometer data multiplied by the mass of the pendulum), (2) lower abdomen longitudinal deflection as measured by uniaxial SPs, (3) upper abdomen longitudinal deflection as measured by triaxial DGSPs, (4) lower chest longitudinal deflection as measured by triaxial CRUX sensors, and (5) upper chest longitudinal deflection as measured by triaxial CRUX sensors.

The test data and FE outputs were digitally filtered using channel frequency class (CFC) filters in accordance with $S A E$ J211-1 Instrumentation for Impact Test [16]. Pendulum 
acceleration and abdomen deflection data were filtered using a CFC 180 filter and chest deflection data were filtered using a CFC 600 filter.

Two techniques were used to validate the abdomen response in the lower and upper abdomen repeatability tests. First, the absolute maximum values for the deflections from the bilateral sensors closest to the impact location were compared with simulations. An agreement window based on the calculated standard deviation (6 samples) between bilateral measurements and 3 repeated tests was used.

An alternative approach to comparing the results was taken by using the CORrelation and Analysis rating software CORAplus v4.0.4 developed by the Partnership for Dummy Technology and Biomechanics (PDB) [17]. The software was used to calculate an overall agreement rating (CORA) between 0 and 1 according to ISO/TS 18571 - Objective Rating Metric for Non-ambiguous Signals [18]. A categorization of the overall rating score is given in Table 2. The following standardized weightings were used: $40 \%$ corridor score $\left(w_{Z}\right), 20 \%$ phase score $\left(w_{P}\right), 20 \%$ magnitude score $\left(w_{M}\right)$, and $20 \%$ slope score $\left(w_{S}\right)$. Data collected from each sensor were time-shifted by the same time increment so that the timing of the peak impactor acceleration aligned with the test data. A manual end time (40 ms) was set for the evaluation interval to account for the slow relaxation of the abdomen foam.

Table 2. Overall CORA Rating Categories

\begin{aligned} & \hline Overall Rating $(C O R A)$ Grade \\ & \hline CORA $>0.94$ Excellent \\ & $0.80<C O R A \leq 0.94$ Good \\ & $0.58<C O R A \leq 0.80$ Fair \\ &$C O R A \leq 0.58$ Poor \\ & \hline\end{aligned}

\section{RESULTS}

Lower Abdomen Repeatability

As mentioned above, Tests \#3, \#7, and \#8 are based on a certification test that is used to measure the biofidelity of the lower abdomen for the THOR-50M. The test setup and FE model are shown in Figure 2. For the peak deflection measurements in the lower abdomen and upper abdomen, the simulation results were within one standard deviation (6 samples) of the test data as shown in Figure 3.

The impactor longitudinal force (Figure 4) received an overall CORA score of 0.893 which is considered "Good.” An unexpected $7 \mathrm{kN}$ peak in force was observed $15 \mathrm{~ms}$ into the simulation. This peak resulted in the overall change in velocity of the pendulum of approximately $1 \mathrm{~m} / \mathrm{s}$ or $16 \%$ higher than expected.

The lower abdomen deflections (Figure 5) received very different CORA scores for the left vs right (0.824 vs 0.538$)$ string potentiometers. This could have been caused by misalignment of the longitudinal axes of the ATD and pendulum in testing or damage to the cable of the left string potentiometer which was identified after testing. In the case of the upper abdomen deflections (Figure 6) the left and right CORA scores were rated as "Excellent."

The lower and upper chest deflections are not shown and were not in agreement with test data because the sensors were far from the impact location and the measured values were relatively small.



Figure 3. Lower Abdomen Pendulum Impact with 25mm Rod (Tests \#3, \#7, \#8); Error Bars Denote Standard Deviation $(n=6)$



Figure 4. Impactor Longitudinal Force (CFC 180)



Figure 5. Lower Abdomen SP Deflection (CFC 180) 




Figure 6. Upper Abdomen DGSP Deflection (CFC 180)

The measured upper and lower abdomen deflections (Figure 5 and Figure 6) were in agreement with the simulation until roughly $40 \mathrm{~ms}$ after impact. This deviation is attributed to the relaxation rates of the abdomen foams not being fully calibrated. For the purpose of this study, the relaxation rates of the foams were not investigated because the point of interest was the peak deflection which is the pass/fail criterion for energy-absorbing table standards.



Figure 7. Impactor Force vs Lower Abdomen SP Deflection

The impactor longitudinal force is plotted against the lower abdomen SP deflection in Figure 7. A stiffer than expected forcedeflection response is observed between 35 and $55 \mathrm{~mm}$ deflection which corresponds with the force spike at $15 \mathrm{~ms}$; however the peak force and peak deflection are in agreement with the test results.

\section{Upper Abdomen Repeatability}

Tests \#4, \#9, and \#10 are based on the upper abdomen certification test for the THOR ATD. The test configuration and corresponding FE model is shown in Figure 8. The peak abdomen deflections were in agreement as shown in Figure 9, but the simulation did not accurately capture the peak chest deflections due to limitations in the dummy positioning. The H3RS FE model had a 15-20 mm gap between the abdomen foam and lower rib that was not observed in testing as seen in Figure 8. The larger spacing caused the impactor's load path to not transfer directly to the lower rib and therefore the lower chest response was not captured.



Figure 8. Upper Abdomen Repeatability Test (Tests \#4, \#9, \#10) Alignment (left) and Corresponding FE Model (right)



Figure 9. Upper Abdomen Pendulum Impact with Steering Wheel (Tests \#4, \#9, \#10); Error Bars Denote Standard Deviation (n=6)

The impactor's peak longitudinal force (Figure 10) was 2 $\mathrm{kN}$ or $18 \%$ lower in the simulation; however, the signal received a CORA rating of 0.944 "Excellent." The change in impactor velocity (not shown) also agreed with test data at approximately $9 \mathrm{~m} / \mathrm{s}$.



Figure 10. Impactor Longitudinal Force (CFC 180) 






- - - - L T\#10 ........ R T\#10 - - - - L T\#4 ….... R T\#4

Figure 11. Lower Abdomen SP Deflection (CFC 180)



Figure 12. Upper Abdomen DGSP Deflection (CFC 180)

As was the case for the previous series of tests, the lower abdomen deflection (Figure 11) CORA scores were different for left vs right (0.823 vs 0.556 ). The right string potentiometer peak deflection was overestimated in the simulation. The upper abdomen deflections (Figure 12) received "Fair" scores. The deflections in the abdomen were in agreement until about $40 \mathrm{~ms}$ after impact, and this deviation is again attributed to the relaxation rates of the abdomen foams.



Figure 13. Lower Chest CRUX Deflection (CFC 600)



Figure 14. Upper Chest CRUX Deflection (CFC 600)



Figure 15. Impactor Force vs Upper Abdomen DGSP Deflection

The lower chest deflections (Figure 13) received a score of "Fair" for the left side and "Good" for the right side. The chest deflections were approximately one standard deviation below what was observed in testing and this difference is attributed to limitations in completely capturing the dummy's initial lumbar positioning.

The overall stiffness of the upper abdomen in the FE model was in good agreement with test data as shown in Figure 15. The peak force is lower than expected but the peak deflection is bounded by the test data.

\section{Lower Abdomen Velocity Sensitivity}

A series of velocity sensitivity tests (Tests \#26, \#22, and \#27) were conducted with a $50.8 \mathrm{~mm}$ round bar weighing $32 \mathrm{~kg}$ at target impact velocities of 5.1, 6.1, and $7.1 \mathrm{~m} / \mathrm{s}$ (respectively) on the lower abdomen at an impact height of SP $+0 \mathrm{~mm}$. The peak impactor forces (Figure 16) were generally in agreement for each case. The lower abdomen deflection (Figure 17) measured in Test \#26 at $5 \mathrm{~m} / \mathrm{s}$ was $25 \%$ higher than expected. This could have been caused by interference from the iliac wings in the pelvis as mentioned in the test report [2]. There was also a larger than expected difference between the left and right string potentiometers as shown in Figure 17 which could have been 
caused by misalignment of the dummy during testing or fraying of the left string potentiometer cable, which was identified after the repeatability tests.



Figure 16. Peak Force vs Impact Speed to Lower Abdomen (Tests \#26, \#22, \#27)



Figure 17. Lower Abdomen SP Peak Deflection vs Impact Speed (Tests \#26, \#22, \#27)

Upper Abdomen Velocity Sensitivity

Another series of velocity sensitivity tests (Tests \#24, \#25, and \#17a) were conducted with a $50 \mathrm{~mm}$ round bar weighing 18 $\mathrm{kg}$ at target impact velocities of 6,7 , and $8 \mathrm{~m} / \mathrm{s}$ (respectively) to the upper abdomen at an impact height of DGSP $+15 \mathrm{~mm}$. The simulations predicted higher peak impactor forces as shown in Figure 18. The upper abdomen peak deflections were in agreement with test data as shown in Figure 19.



Figure 18. Peak Force vs Impact Speed to Upper Abdomen (Tests \#24, \#25, \#17a)



Figure 19. Upper Abdomen DGSP Peak Deflection vs Impact Speed (Tests \#24, \#25, \#17a)

The simulations were within 0.3 to $0.6 \mathrm{~m} / \mathrm{s}$ or 5 to $10 \%$ of the measured change in velocity from the tests. As was the case with the upper abdomen steering wheel impact simulation, the lower chest deflection was underestimated due to limitations with initial positioning of the lumbar.

\section{Lower Abdomen Height Sensitivity}

The lower abdomen was impacted with a $50.8 \mathrm{~mm} 32 \mathrm{~kg}$ round rod at $6.1 \mathrm{~m} / \mathrm{s}$ at an impact height of SP $+0 \mathrm{~mm}$ (Test \#22) and at a height of SP $+25 \mathrm{~mm}$ (Test \#23). The peak impact forces from the tests and simulations were in agreement between as shown in Figure 20. The lower abdomen SP peak deflection test results were unexpectedly lower at the $\mathrm{SP}+0 \mathrm{~mm}$ height when compared to SP $+25 \mathrm{~mm}$ as shown in Figure 21. This was likely due to interference from the iliac wings in the pelvis of the H3$\mathrm{RS}$ as mentioned in the test report. 




Figure 20. Peak Force vs Impact Height from String Potentiometer (Tests \#22, \#23)

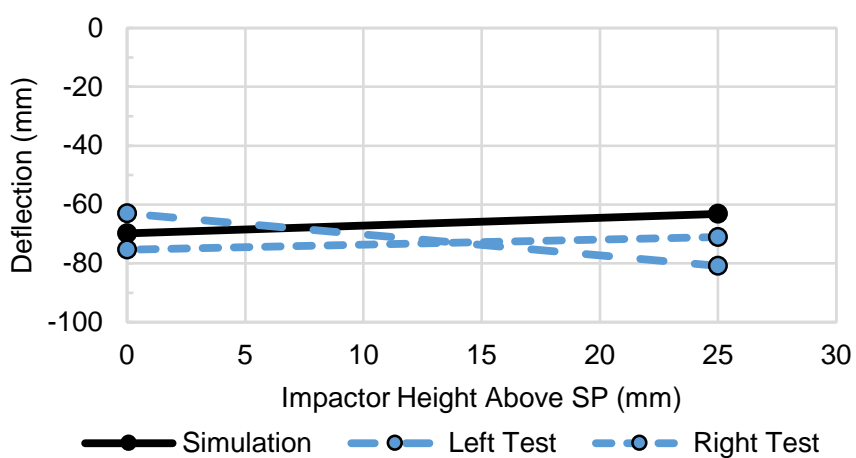

Figure 21. Lower abdomen SP Peak Deflection vs Impact Height from SP (Tests \#22, \#23)

\section{Upper Abdomen Height Sensitivity}

Lastly, a series of height sensitivity tests (Tests \#21, \#16, $\# 17 \mathrm{a}$, and \#18) was conducted on the upper abdomen at heights of $-10,0,15$, and $40 \mathrm{~mm}$ (respectively) from the DGSP sensors. The target impact velocity was $8 \mathrm{~m} / \mathrm{s}$ with a $50 \mathrm{~mm} 18 \mathrm{~kg}$ round rod pendulum impactor. The simulations resulted in an underestimated peak force at DGSP $-10 \mathrm{~mm}$ and an overestimated peak force at DGSP $+40 \mathrm{~mm}$ when compared with test data while the peak forces were generally in agreement at DGSP +0 and DGSP $+15 \mathrm{~mm}$ as seen in Figure 22. The upper abdomen DGSP peak deflections were generally in agreement except for the SP $-10 \mathrm{~mm}$ case which was underestimated by 14\% as seen in Figure 23.

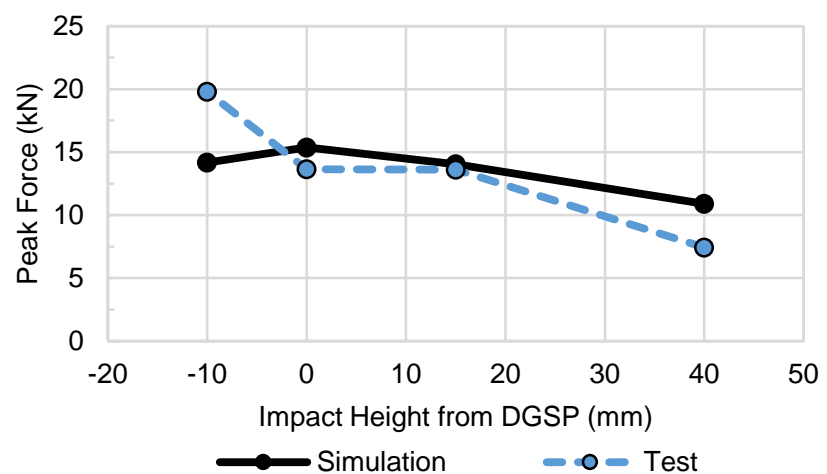

Figure 22. Peak force vs impact height from DGSP (Tests \#21, \#16, \#17a, \#18)



Figure 23. Upper Abdomen DGSP Peak Deflection vs Impact Height from DGSP (Tests \#21, \#16, \#17a, \#18)

\section{DISCUSSION}

Several of the simulations did not capture the deflections in the lower abdomen string potentiometers. It is likely that the iliac wings in the pelvis of the H3-RS interfered with some of the lower abdomen pendulum impact tests. This effect is also sometimes observed in the THOR-50M which has a similar iliac wing configuration.

The H3-RS was not perfectly aligned with the longitudinal axis of the pendulum impactor in the tests. This misalignment resulted in disagreement between the left and right bilateral deflection sensors and was not captured in the simulations. An example of the misalignment can be seen in Test \#4 (Figure 15) where the left and right upper abdomen DGSPs had peak deflections with a difference of roughly $20 \mathrm{~mm}$.

It was found that the overall score calculated using ISO/TS 18571 was overly sensitive to a disagreement in phase. For the purposes of this study the authors were most interested in the magnitude score $\left(w_{M}\right)$ because peak chest and abdomen deflections are used as pass/fail requirements in energyabsorbing table standards. The magnitude score only accounted for $20 \%$ of the overall score while the corridor score $\left(w_{z}\right)$ and the phase score $\left(w_{P}\right)$ combined to total $60 \%$ of the overall score and were both very sensitive to phase. Therefore, the authors did not rely solely on the CORA score to validate the abdomen response. 
Further calibration of the foam relaxation rate in the abdomen could improve the settling behavior of the sensors. However, the peak internal deflection measurements have been shown to be in the range of repeatability for pendulum impact tests. It is therefore concluded that the H3-RS FE model is capable of producing valid results for impacts to the lower and upper abdomen.

This paper is intended to accompany a companion paper [3] studying the behavior of the H3-50M and H3-RS FE models in a dynamic sled test of an energy-absorbing table design. This work is also intended to demonstrate that the thoracic response of the H3-RS FE model is validated for research and development purposes but not for demonstrating compliance in lieu of dynamic sled testing of an energy-absorbing table. Further analyses of the APTA table sled test will permit evaluation of the testing environment with the goal of improving passenger rail safety through modernized safety standards.

\section{ACKNOWLEDGMENTS}

This work was performed under the FRA Office of Research, Development, and Technology Equipment Safety Research Program. The authors would like to thank the program manager Jeffrey Gordon. The authors would also like to thank Dr. Hubert Ley and Dr. Cezary Bojanowski at Transportation Research and Analysis Computing Center (TRACC) for access to their high performance computing cluster and Dr. Stephen Summers at NHTSA for access to LS-DYNA. Lastly, the authors would like to thank Marine Favre-Decloux and Michael Pittman at TRL for training in the use of the H3-RS FE model and arranging for the loan of the model respectively.

\section{REFERENCES}

[1] The Rail Safety and Standards Board, "Hybrid III Rail Dummy specification (T066 Report)," January 2008.

[2] D. Hynd and J. A. Carrol, "Abdomen Impact Testing of the Hybrid III Rail Safety (H3-RS) Anthropomorphic Test," DOT/FRA/ORD-17/17, September 2017.

[3] S. Eshraghi, K. Severson, D. Hynd and A. B. Perlman, "Finite Element Analysis of the Passenger Rail Equipment Workstation Table Sled Test," in ASME IMECE, Pittsburgh, PA, 2018.

[4] American Public Transportation Association, "Fixed Workstation Tables in Passenger Rail Cars," APTA PRCS-S-018-13, Rev. 1, October 2015.

[5] National Transportation Safety Board, "Collision of Burlington Northern Santa Fe Freight Train with Metrolink Passenger Train Placentia, California, April 23, 2002," Railroad Accident Report NTSB/RAR-03/04, Washginton, D.C., October 2003.
[6] D. Parent, D. Tyrell and A. B. Perlman, "Crashworthiness Analysis of the Placentia, CA Rail Collision," in International Crashworthiness Conference, San Francisco, CA, 2004.

[7] D. Parent, D. Tyrell, K. Jacobsen and K. Severson, "Crashworthiness Analysis of the January 26, 2005 Glendale, California Rail Collision," in ASME Joint Rail Conference, Paper No. JRC2011-56132, March 2011.

[8] Dutch Safety Board, "Train Collision Amsterdam Westerpark," The Hague, December 2012.

[9] Rail Safety Standards Board, "Requirements for Rail Vehicle Structures," GM/RT2100 Issue Five, June 2012.

[10] J. Cavanaugh, G. Nyquist, S. Goldberg and A. King, "Lower Abdominal Tolerance and Response," in 30th Stapp Car Crash Conference, Paper no. 861878, October 1986.

[11] G. Nusholtz and P. Kaiker, "Abdominal Response to Steering Wheel Loading," in 14th International Technical Conference on the Enhanced Safety of Vehicles (ESV), Paper no. 94-S1-O-05, 1994.

[12] M. B. Panzer, S. Guidice and D. Parent, "THOR 50th Male Finite Element Model User Manual Model Version 2.1 for LS-Dyna," US Department of Transportation, National Highway Traffic Safety Administration, January 2015.

[13] "LS-DYNA," Livermore Software Technology Corporation, [Online]. Available: http://www.lstc.com/. [Accessed 30 April 2018].

[14] S. Guha, "LSTC_NCAC Hybrid III 50th Dummy Positioning \& Post-Processing," LSTC \& NCAC, May 29, 2014.

[15] National Highway Traffic Safety Administration , "Biomechanics Test Database," [Online]. Available: https://wwwnrd.nhtsa.dot.gov/database/bio/information.htm. [Accessed 29 April 2018].

[16] SAE J211-1, "Instrumentation for Impact Test," Warrendale, PA, Revised March 1995.

[17] C. Thunert, "CORAplus Release 4.0.4 User's Manual," Partnership for Dummy Technology and Biomechanics, Ingolstadt, Germany, 2017.

[18] ISO/TS 18571:2014(E), "Road vehicles - Objective rating metric for non-ambiguous signals," International Organization for Standardization, Geneva, Switzerland, 2014.

[19] S. Guha, "50th Percentile H-III," LSTC \& NCAC, December 2015. [Online]. Available: http://www.lstc.com/download/dummy_models. 